\title{
Channel Alternation And Rotation For Tri-sectored Directional Antenna Cellular Systems
}

\author{
Vincent A. Nguyen, Peng-Jun Wan, and Ophir Frieder \\ Computer Science Department \\ Illinois Institute of Technology \\ Chicago, Illinois \\ vnguyen@iit.edu,wan@cs.iit.edu, and ophir@ir.iit.edu
}

\begin{abstract}
Due to discrete reuse cluster sizes, disjoined and uniformed channel assignment, conventional tri-sectored cellular systems have not taken full advantage of antenna directivities. In this paper, we present a novel Channel Alternation and Rotation (CAR) scheme to coordinate channel assignment with antenna directivities. In CAR, cell layout is based on two-tier cell-reuse structure and each cell is allocated one extra channel set for channel alternations and rotations. The extra channel set gives network designer the flexibility to assign channels according to nearest front lobe interference avoidant strategy to enhance co-channel interference ratio (C/I). CAR allows deployment of smaller, non-integer reuse cluster sizes based on $\mathrm{C} / \mathrm{I}$ requirements, thus increases frequency reuse efficiency. CAR reuse plans can increase channel capacity up to $31.25 \%$ while still maintain comparable $\mathrm{C} / \mathrm{I}$ margins. CAR is simple and can be employed in any existing directional antenna system. Therefore, it truly does not impose any additional cost.
\end{abstract}

\section{INTRODUCTION}

Personal Communication System (PCS) is driven mainly by frequency reuse efficiency, Quality Of Service (QOS), and low infrastructure costs. Frequency reuse efficiency in a cellular network is limited by co-channel interference, which directly determines the system capacity and QOS. Thus, to maximize system capacity, cellular network designer must strive to reuse the scarce radio resource efficiently.

Currently, most conventional cellular systems employ three $100^{\circ}$ to $120^{\circ}$ directional antennas at each base station (BS) in reuse clusters of 3,4 , or 7 cells [1][2]. Based on fixed channel assignment scheme, 3 disjoined channel sets are assigned to each BS and repeated uniformly in all other clusters to provide equidistant separation among co-channel cells. However, with those fixed constraints, conventional reuse plans still have not taken full advantages of antenna directivities to maximize frequency reuse efficiency.

Sector rotation schemes based on group reuse and interleaved channel assignment and two-site reuse scheme have been proposed in [1][2][3][7]. However, they either employ four $90^{\circ}$, three $60^{\circ}$ to $70^{\circ}$, or six $60^{\circ}$ directional antennas. Although these schemes improve $\mathrm{C} / \mathrm{I}$ and capacity, they are costly to switch from the current $100^{\circ}$ to $120^{\circ}$ trisectored antenna systems, since antenna replacement is needed system-wide. They, however, have been deployed in some newer systems, more than in existing tri-sectored system [1].

In this paper, we present a novel CAR scheme to coordinate channel assignment with antenna directivities. In
CAR, cell layout is based on two-tier cell-reuse structure and each cell type is allocated one extra channel set to be used for channel alternations and rotations. This extra channel set allows channels to be assigned according to nearest front lobe interference avoidant strategy to enhance co-channel interference ratio $(\mathrm{C} / \mathrm{I})$. CAR allows deployment of smaller, non-integer reuse cluster sizes based on $\mathrm{C} / \mathrm{I}$ requirement, thus increases frequency reuse efficiency. The performance analysis shows that CAR reuse plans can increase channel capacity up to $31.25 \%$ while still maintain comparable $\mathrm{C} / \mathrm{I}$ protection margins in comparison with the targeted conventional reuse plans.

CAR scheme relaxes the constraints assumed in conventional reuse plans thus provides cellular network designer the flexibility to deploy unconventional reuse cluster sizes and rnultiple reuse distances based on $\mathrm{C} / \mathrm{I}$ rather than predetermined cluster sizes as in conventional system. CAR is simple and can be implemented in any existing directional antenna system, thus it truly does not impose any additional cost.

The renainder of this paper is organized as follows. Section II further describes frequency reuse planning in conventional directional antenna systems. Section III describes how directional antenna systems are exploited in CAR, and presents the CAR scheme. In Section IV, we demonstratie the performance advantages of CAR approach over conventional reuse plans based on system capacity and $\mathrm{C} / \mathrm{I}$ margins. Finally, Section $\mathrm{V}$ concludes this paper.

\section{Tri-SleCtORED DiRECTIONAL ANTENNA SySTEMS}

In a cellular network, the entire available spectrum is partitioned into channel sets and assigned to each cluster of $N$ cells. To provide equidistant co-channel separations, $N$ must be a rhombic number determined by the two shift parameters $i$ and $j$, as expressed in

$$
N=i^{2}+i j+j^{2}
$$

Thus, $N$ is restricted within a finite set of values e.g. 3,4,7. Furthermore, each sector in a cell is assigned a set of channels uniquely different from all other sectors in the cluster and repeated uniformly system-wide. The equidistant separation allows the same set of channels to be used simultaneously in all clusters at all times. Shorter reuse distance or: smaller $N$ increases frequency reuse efficiency 
which directly determines the system capacity but decreases $\mathrm{C} / \mathrm{I}$, which affects the QOS. Longer reuse distance or bigger $N$ improves C/I, but reduces system capacity.

Different cellular systems require different $\mathrm{C} / \mathrm{I}$ thresholds. As a general guidance, $18 \mathrm{~dB}, 14 \mathrm{~dB}$, and $9 \mathrm{~dB}$ are the minimum acceptable $\mathrm{C} / \mathrm{I}$ margins in Advanced Mobile Phone System (AMPS), digital Time Division Multiple Access (TDMA) such as IS-136, and Global System for Mobile Communication (GSM), respectively. Normally $18 \mathrm{~dB}$ can be maintained with $N=7$ utilizing omni-directional antenna system. However, currently most conventional cellular systems employ three $100^{\circ}$ to $120^{\circ}$ directional antennas at each BS in clusters of 3,4 , and 7 cells [1][2][8]. Directional system is thus denoted $N^{*} k$ reuse plan, where $k$ is the number of sectors in a cell.

Unlike omni-directional antenna which power radiates equally in all directions, tri-sectored cellular system uses 3 directional antennas at each BS that direct main beam power on to the 3 front lobe areas. Fig. 1 depicts a typical $120^{\circ}$ antenna radiation pattern obtained at [10]. Within which and based on signal strength as intended, front lobe region is generally within azimuth $\theta=0^{0}$ to $\pm 60^{\circ}$, side lobe is from $\pm 60^{\circ}$ to $\pm 120^{\circ}$, and back lobe is from $\pm 120^{\circ}$. This radiation pattern is also used to compute $\mathrm{C} / \mathrm{I}$ in this paper.

In conventional $7 \times 3$ reuse plan depicted in Fig. 2, seven cells, A, B, C, D, E, F, and G, are grouped into a cluster and assigned such that all co-channel cells (of the same type) are equidistant apart. Each cell has 3 sectors and is assigned 3 disjoined channel sets. Thus a total of 21 channel sets are used and typically assigned as follows: $\mathrm{A}=\{1,8,15\}, \mathrm{B}$ $=\{2,9,16\}, \ldots$ and $G=\{7,14,21\}$. Fig. 2 also depicts the worse interference scenario, that is, when MS is at the fringe of a sector e.g. sector 1 (channel 1 ) in cell $A_{0}$. For simplicity, only first tier adjacent co-channel interferers $A_{1}$ to $A_{6}$ are labeled. Among them, only $A_{4}$ and $A_{5}$ are from the antenna front lobes while $A_{1}, A_{2}, A_{3}$, and $A_{6}$ are side and back lobe interferers. In comparison with omni-directional system, $7 \times 3$ reuse plan increases $\mathrm{C} / \mathrm{I}$ to $20.9 \mathrm{~dB}$ from $17.8 \mathrm{~dB}$. This improvement is due mainly to the reduction of interference from the two side lobe interferers and the negligible interference from the two back lobe co-channels.

The $4 \times 3$ and $3 \times 3$ reuse plans have also been widely deployed likewise in IS-136, PDC and GSM systems, particularly in Japan and Europe, respectively [9]. Reuse plan tighter than $3 \times 3$, e.g. $2 \times 3$, in $100^{\circ}$ to $120^{\circ}$ tri-sectored cellular system is impractical, since co-channel cells are adjoined and reducing the separation between co-channel cells hence reduces $\mathrm{C} / \mathrm{I}[1][7][8]$.

\section{Channel Alternation And Rotation}

\section{A. Conceptual Design}

Since interference from antenna back lobe is negligible and interference from side lobe is significantly reduced, CAR is proposed to take full advantages of antenna directivities by

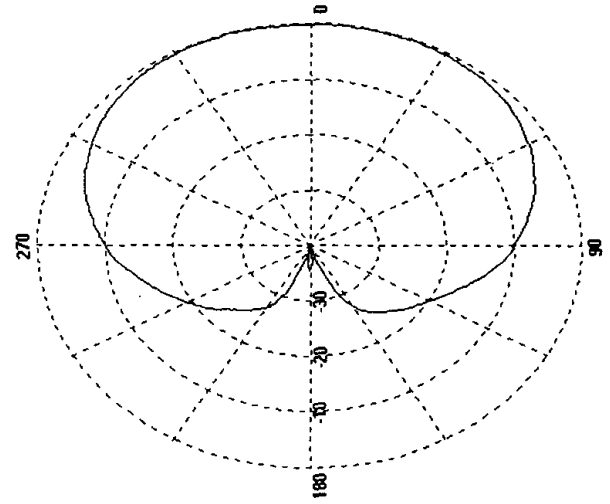

Fig. 1. $120^{\circ}$ directional antenna pattern

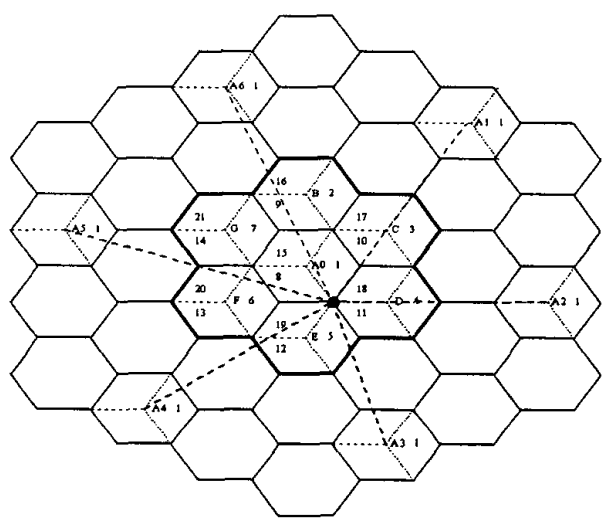

Fig. 2. $7 \times 3$ reuse plan and worse interference scenario

systematically alternating and rotating channels to minimize the effects of and to avoid front lobe interference to and from the nearest co-channel BS to enhance $\mathrm{C} / \mathrm{I}$, thus increases system capacity. To achieve those objectives, we employ:

i. Cell Layout Planning: Two-tier cell-reuse separations.

ii. Frequency Planning: Nearest front lobe interference avoidant strategy

In cell layout planning (i): First, two-tier cell-reuse separations provides network designer the flexibility to control $(N)$ and deploy reuse plans based on $\mathrm{C} / \mathrm{I}$ requirement, rather than being restricted within $3,4,7$ set by (1). Secondly, this cell layout minimizes interference to multiple nearest and equidistant co-channels at its strongest power.

In frequency planning (ii): First, when antenna main beam power radiates toward the nearest co-channel cell entirely, interfered channel can be alternated, thus front lobe interference is avoided. Secondly, if main beam power projects toward nearest co-channel cell partially, interfered channel can be rotated, thus interference is minimized, since it becomes side lobe interferer instead. $\mathrm{C} / \mathrm{I}$ is then the result of interference from antenna side and back lobes and from 
antenna front lobes of co-channels that are farther away, neither of which has significant impact on $\mathrm{C} / \mathrm{I}$ in comparison with nearest front lobe interference, if existed.

\section{B. Cell Reuse Structure}

In CAR, cells are labeled sequentially from A to $N$ (type) in zigzag order along each pair of interlocking columns and repeated likewise in adjacent pairs. This technique produces two-tier cell-reuse structure which co-channel cells are separated by 1 column and $N-1$ interlocking rows. In this paper, we limit $\mathrm{N}$ to 2,3,4, and 5. Fig. 3 depicts cell layouts for $\mathrm{N}=\{2,4\}$ as described above. When $\mathrm{N}=3$ (not shown), the cell structure is identical to conventional counterpart; however, the difference is seen in $\mathrm{N}=4$ (and 5). $\mathrm{N}=2$ is not used in conventional system due to adjoined co-channel cells.

Let's consider the $\mathrm{N}=4$ cell layout depicted in Fig. 3. Assume $A_{0}$ is the center cell and, in clockwise direction, $A_{1}$ to $A_{8}$ are co-channel cells starting from the top right. Also given that $\mathrm{f} 90^{\circ}, \mathrm{f} 210^{\circ}$, and $\mathrm{f} 330^{\circ}$ are functions of degrees indicating the main beam directions of the three sectors (at each BS), as denoted in Fig. 3. If the channel used in 190 of $\mathrm{A}_{0}$ is alternated in $\mathrm{A}_{2}$, front lobe interference to nearest cochannel is avoided. Interference to $A_{1}$ and $A_{3}$ is already reduced due mainly to longer reuse distance. Next, let's consider sector $\mathrm{f} 330$ in $\mathrm{A}_{0}$, if $\mathrm{f} 210$ and $\mathrm{f} 330$ in $\mathrm{A}_{6}$ are rotated, f330 of $A_{0}$ becomes side lobe interferer to $f 210$ of $A_{6}$ hence interference is reduced. $\mathrm{F} 330$ also projects toward cell $\mathrm{A}_{8}$ entirely, yet, if the channel is alternated, then interference is avoided. $\mathrm{F} 210$ in $\mathrm{A}_{0}$ is also arranged likewise.

\section{Channel Assignment}

In CAR, each cell type is allocated one extra channel set for channel alternation that results in $3+1$ channel sets per cell type and $N(3+1)$ sets system-wide. Thus, CAR can be generalized as $N(k+x)$ reuse plan. Since each cell is assigned only 3 out of 4 allocated sets, there are $\left(\begin{array}{l}4 \\ 3\end{array}\right)=4$ unique patterns per cell type. For example, in $4 x(3+1)$ reuse plan, the channels allocated to cells of type $A=\{1,5,9,13\}$. Thus, type A cell consists of patterns: $A_{p 1}=\{1,5,9\}, A_{p 2}=$ $\{1,5,13\}, A_{p 3}=\{1,9,13\}$, and $A_{p 4}=\{5,9,13\}$ where $p i$ indexes the pattern number. With respect to $N$, channel allocation and assignment patterns are illustrated in Table $\mathrm{I}$.

Although $k$ and $x$ can have different values, in the following algorithm, we limit $k$ to 3 and $x$ to 1 for use in $100^{\circ}$ to $120^{\circ}$ directional antenna systems.

1. Choose the cell layout and channel allocation and pattern as previously described for the proper reduced size $N=N-\beta \geq 2$, where $\beta$ is the reduction factor.

2. For each cell-type $\mathrm{I}$ in $\mathrm{N} ; \mathrm{I}=\mathrm{A}$ to $\mathrm{N}$

a) Label 4 channel sets allocated to type I cell shown in Table I as $\left\{\mathrm{C}_{1}, \mathrm{C}_{2}, \mathrm{C}_{3}, \mathrm{C}_{4}\right\}$

b) Start from the left most interlocking columns of the cell grid for first cell of type I cell,

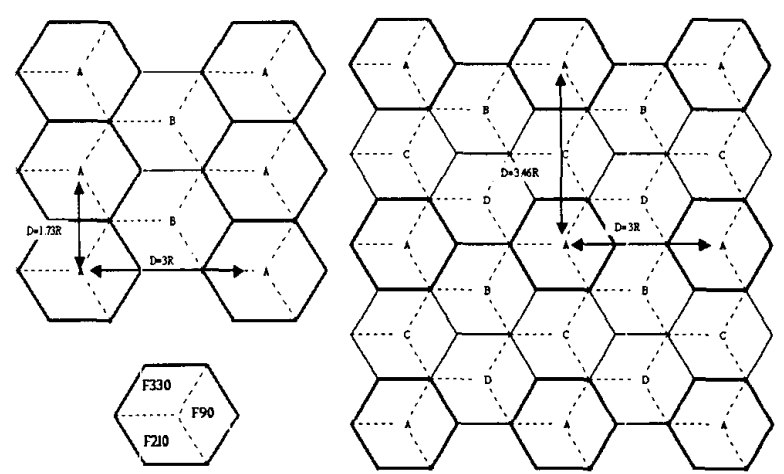

Fig. 3. CA.R cell structure for $N=2$ (left), $N=4$ (right) and sector orientation (bottom left)

TABLE I

Channel allocation In CaR Reuse Plans

\begin{tabular}{|c|c|c|c|c|}
\hline $\begin{array}{l}\text { Reuse Plan } \\
\text { Cell Type }\end{array}$ & $5 \times(3+1)$ & $4 \times(3+1)$ & $3 \times(3+1)$ & $2 \times(3+1)$ \\
\hline $\mathrm{A}$ & $1,6,11,16$ & $1,5,9,13$ & $1,4,7,10$ & $1,3,5,7$ \\
\hline Pattern $A_{p l}$ & $1,6,11$ & $1,5,9$ & $1,4,7$ & $1,3,5$ \\
\hline$A_{\mathrm{D} 2}$ & $1,6,16$ & $1,5,13$ & $1,4,10$ & $1,3,7$ \\
\hline$A_{p 3}$ & $1, \overline{11}, 16$ & $\overline{1,9,13}$ & $1,7,10$ & $1,5,7$ \\
\hline $\mathrm{A}_{\mathrm{p} 4}$ & $6,11,16$ & $5,9,13$ & $4,7,10$ & $3,5,7$ \\
\hline $\mathrm{B}$ & $2,7,12,17$ & $2,6,10,14$ & $2,5,8,11$ & $2,4,6,8$ \\
\hline $\mathrm{B}_{\mathrm{pl}}$ & $2,7,12$ & $2,6,10$ & $2,5, \overline{8}$ & $2,4,6$ \\
\hline $\mathrm{B}_{\mathrm{p} 2}$ & $2,7,17$ & $2,6,14$ & $2,5,11$ & $2,4,8$ \\
\hline $\mathrm{B}_{\mathrm{p} 3}$ & $2,12,17$ & $2,10,14$ & $2,8,11$ & $2,6,8$ \\
\hline $\mathrm{B}_{\mathrm{p} 4}$ & $7,12,17$ & $6, \overline{10,14}$ & $5,8,11$ & $4,6,8$ \\
\hline $\mathrm{C}$ & $3,8,13,18$ & $3,7,11,15$ & $3,6,9,12$ & \\
\hline $\mathrm{C}_{\mathrm{pl}}$ & $3,8,13$ & $3,7,11$ & $3,6,9$ & \\
\hline $\mathrm{C}_{\mathrm{p} 2}$ & $3,8,18$ & $3,7,15$ & $3,6,12$ & \\
\hline$C_{p 3}$ & $3,13,18$ & $3,11,15$ & $3,9,12$ & \\
\hline $\mathrm{C}_{\mathrm{p} 4}$ & $8,13,18$ & $7,11,15$ & $6,9,12$ & \\
\hline D & $4,9,14,19$ & $4,8,12,16$ & & \\
\hline $\mathrm{D}_{\mathrm{p} I}$ & $4,9,14$ & $4,8,12$ & & \\
\hline $\mathrm{D}_{\mathrm{p} 2}$ & $4,9,19$ & $4,8,16$ & & \\
\hline$D_{\mathrm{p} 3}$ & $4,14,19$ & $4,12,16$ & & \\
\hline$\Sigma_{\mathrm{p} 4}$ & $9,14,19$ & $8,12,16$ & & \\
\hline$E$ & $5,10,15,20$ & & & \\
\hline $\mathrm{E}_{\mathrm{p} 1}$ & $5,10,15$ & & & \\
\hline $\mathrm{F}_{p 2}$ & $5,10,20$ & & & \\
\hline $\mathbf{E}_{\mathrm{p} 3}$ & $5,15,20$ & & & \\
\hline $\mathrm{E}_{\mathrm{p} 4}$ & $10,15,20$ & & & \\
\hline
\end{tabular}

- Assign $C_{1}$ to sector $f 90, C_{2}$ to sector $f 210$, and $C_{3}$ to sector $\mathrm{f} 330$. Thus, $\mathrm{C}_{1}$ and $\mathrm{C}_{4}$ become alternating channel pair (AP) and $\mathrm{C}_{2}$ and $\mathrm{C}_{3}$ become rotating channel pair (RP).

" For every co-channel cell on the same row, rotate the RP and assign to $f 210$ and $f 330$, respectively. Assign previously unused AP channel to 990 .

c) For each remaining rows of type I cells

- Reverse the AP and RP roles. Thus: AP become 
$\mathrm{RP}$ and vice versa.

- Move to the first cell on the far left:

- Choose from RP the unused channel in the previous nearest row-adjacent co-channel cell where $\mathrm{f} 330$ is pointing to, assign that channel to f330. Assign the other RP channel to $\mathrm{f} 210$.

- From the top corner of 990 , identify the unused AP channel in nearest $\mathrm{f} 210$ of the two adjacent co-channel cells on previous co-channel row. Assign it to $\mathrm{f} 90$.

- For each co-channel cell on the same row, rotate the RP and assign to $\mathrm{f} 210$ and $\mathrm{f} 330$, respectively. Assign previously unused AP channel to 90 .

Applying the above algorithm, for cell structures $N=\{2,3$, 4 , and 5$\}$, we obtain the channel assignments and repeating patterns for $2 \times(3+1), 3 \times(3+1), 4 \times(3+1)$, and $5 \times(3+1)$ reuse plans. Fig. $4 a-b$ depicted reuse plans for $\mathrm{N}=\{2$ and 4$\}$.

\section{Performance evaluation}

\section{A. Reuse Factor}

In conventional system, each channel set is used once in the cluster, therefore cluster of $N$ cell is also the reuse factor. In CAR, each channel set is reused 3 times in repeating pattern of $N(k+x)$ cells as depicted in Fig. 4. Thus, the reuse factor for CAR labeled $N_{c a r}$ can be generalized as:

$$
N_{c a r}=\frac{N(k+x)}{j}
$$

where $j$ is the number of times the same channel set is repeated in the pattern. Hence, $N_{c a r}$ for $2 \times(3+1), 3 \times(3+1)$, $4 \times(3+1)$, and $5 \times(3+1)$ are $2.6,4,5.3$, and 6.6 , respectively.

\section{B. C/I and System Capacity}

To illustrate the performance of CAR against conventional reuse plans, worse interference scenario -when the user is at the fringe of a serving sector- is assumed and expressed as,

$$
\frac{C}{I}=10 \log \left[\frac{G\left(\theta_{0}\right)}{\sum_{i=1}^{n} G\left(\theta_{i}\right)\left(\frac{D_{i}}{R}\right)^{-y}}\right]
$$

where $\mathrm{R}$ is the radius of the cell, normalized to 1 ; thus $D_{i} / R$ (or $d_{i}$ ) represents the normalized distance from MS to $i^{\text {th }}$ co-channel BS; $n$ is the number of co-channel interferers, $\gamma$ is the path loss exponent set equal 4; and $G\left(\theta_{0}\right)$ and $G\left(\theta_{i}\right)$ are antenna gains by MS from the serving BS and $i^{\text {th }}$ cochannel BS at angle $\theta_{i}$, from antenna bore-sight (at $0^{0}$ ) respectively, and expressed in decibels as,
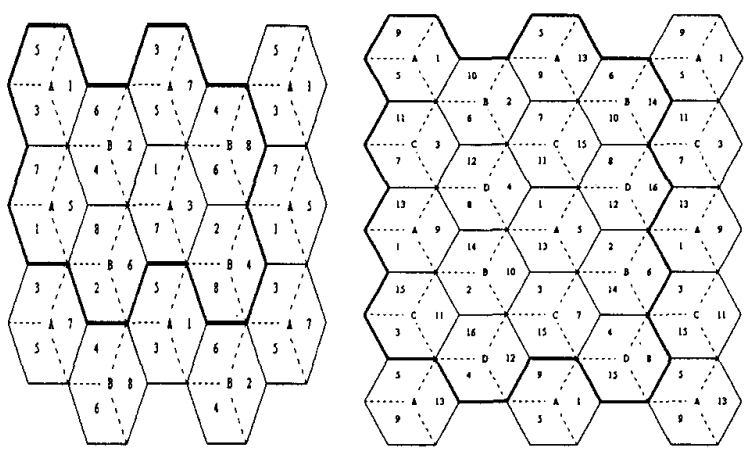

Fig 4. $2 \times(3+1)$ (left) and $4 \times(3+1)$ reuse pattern (right)
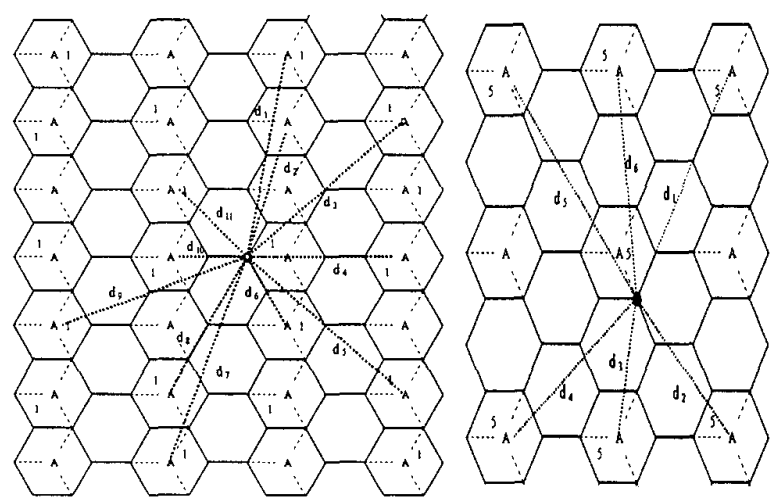

Fig. 5. Worse interference in $2 \times(3+1)$ (left) and $4 \times(3+1)$ (right)

$$
G\left(\theta_{i}\right)=10^{G\left(\theta_{i}\right)_{d B} / 10}
$$

In our analyses, we assume that cell sites are equal and transmit at the same power. We include all first tier cochannel interferers and also consider all front lobe and side lobe interferers from second tier co-channels which interference may be significant, generally when $d_{i}<7 \mathrm{R}$ and $\theta_{i}<90^{\circ}$. In this study, we neglect antenna down tilting and shadow fading since they are mutually independent, and can offset each other gain and loss, respectively. Using (3), (4), and $120^{\circ}$ directional antenna radiation pattern shown in Fig. 1 , we computed $\mathrm{C} / \mathrm{I}$ of worse locations for all reuse plans. Table II and III show the results for worse cases depicted in Fig. 5. Tables IV to VI summarize and compare the performance of CAR against targeted conventional plans.

Table IV provides worse $C / I$ in $4 \times(3+1)$ and $5 \times(3+1)$ reuse plans, which are at $17.8 \mathrm{~dB}$ and $19.3 \mathrm{~dB}$ respectively. These $\mathrm{C} / \mathrm{I}$ are at and above the acceptable margins required by AMPS. To provide $18 \mathrm{~dB}$, conventional system must employ $N=7$. Thus, $4 \times(3+1)$ and $5 \times(3+1)$ increase channel capacity by $31.25 \%$ and $5 \%$ over $7 \times 3$ plan, respectively, due mainly to smaller reuse factor $\left(N_{c a r}=5.3\right.$ and 6.6). 
TABLE II

Worse C/I In $2 x(3+1)$ Reuse Plan

\begin{tabular}{|c|c|c|c|c|}
\hline & $D_{i} / R$ & $\theta_{i}$ & $G\left(\theta_{i}\right)_{d B}$ & $G\left(\theta_{i}\right)\left(D_{i} / R\right)^{-y}$ \\
\hline$d_{0}$ & 1 & 60 & -2.9 & $5.13 \mathrm{E}-01$ \\
\hline$d_{1}$ & 5.3 & 101 & -10.9 & $1.04 \mathrm{E}-04$ \\
\hline $\mathrm{d}_{2}$ & 3.6 & 346 & -0.1 & $5.78 \mathrm{E}-03$ \\
\hline $\mathrm{d}_{3}$ & 5.3 & 259 & -10.0 & $1.28 \mathrm{E}-04$ \\
\hline$d_{4}$ & 4.0 & 60 & -3.0 & $1.96 \mathrm{E}-03$ \\
\hline$d_{5}$ & 5.3 & 259 & -10.0 & $1.28 \mathrm{E}-04$ \\
\hline$d_{6}$ & 2.0 & 240 & -13.3 & $2.92 \mathrm{E}-03$ \\
\hline $\mathrm{d}_{7}$ & 5.4 & 286 & -5.5 & $3.29 \mathrm{E}-04$ \\
\hline $\mathrm{d}_{8}$ & 4.0 & 60 & -3.0 & $1.96 \mathrm{E}-03$ \\
\hline $\mathrm{d}_{9}$ & 5.3 & 341 & -0.2 & $1.22 \mathrm{E}-03$ \\
\hline $\mathrm{d}_{10}$ & 2 & 240 & -13.3 & $2.92 \mathrm{E}-03$ \\
\hline$d_{11}$ & 2.6 & 41 & -1 & $1.62 \mathrm{E}-02$ \\
\hline $\mathrm{C} / \mathrm{I}$ & & & & $11.8 \mathrm{~dB}$ \\
\hline
\end{tabular}

TABLE III

Worse C/I In 4x(3+1) Reuse Plan

\begin{tabular}{|c|c|c|c|c|}
\hline & $D_{i} / R$ & $\theta_{i}$ & $G\left(\theta_{i}\right)_{d B}$ & $G\left(\theta_{i}\right)\left(D_{i} / R\right)^{-y}$ \\
\hline $\mathrm{d}_{0}$ & 1 & 60 & -3.0 & $5.01 \mathrm{E}-01$ \\
\hline$d_{1}$ & 5 & 0 & 0 & $1.60 \mathrm{E}-03$ \\
\hline $\mathrm{d}_{2}$ & 3.6 & 346 & -0.1 & $5.78 \mathrm{E}-03$ \\
\hline $\mathrm{d}_{3}$ & 2.6 & 161 & -28.4 & $2.95 \mathrm{E}-05$ \\
\hline$d_{4}$ & 4.4 & 83 & $-7 . \overline{7}$ & $4.70 \mathrm{E}-04$ \\
\hline$d_{5}$ & 5.6 & 291 & -4.5 & $3.69 \mathrm{E}-04$ \\
\hline$d_{6}$ & 4.4 & 203 & -23.5 & $1.24 \mathrm{E}-05$ \\
\hline $\mathrm{C} / \mathrm{I}$ & & & & $17.8 \mathrm{~dB}$ \\
\hline
\end{tabular}

Table $\mathrm{V}$ compares the $3 \times(3+1)$ with conventional $4 \times 3$ reuse plan. Since the numbers of channel sets are equal, as both use 12 channel sets system-wide, channel capacity remains the same as expected.

Table VI shows that $2 \times(3+1)$ plan provides a comparable $\mathrm{C} / \mathrm{I}$ margin, $11.8 \mathrm{~dB}$ vs. $12.3 \mathrm{~dB}$ in worse case. Due to smaller reuse factor, $N_{\text {car }}=2.6$ vs. $N=3,2 \times(3+1)$ reuse plan increases channel capacity by $12.50 \%$ over $3 \times 3$ plan. However, with $\mathrm{N}=2$, co-channel cells are adjoined, thus antenna with frontto-back ratio $\geq 25 \mathrm{~dB}$ is used to take full advantage of CAR.

\section{CONCLUSION}

In this paper, we presented an innovative channel assignment scheme, namely Channel Alternation and Rotation. CAR is based on two-tier cell-reuse separation structure. Also each cell is allocated one extra channel set to provide network designer the flexibility to alternate and rotate channels according to nearest front lobe interference avoidant strategy based on antenna directivities to enhance $\mathrm{C} / \mathrm{I}$. CAR provides multiple channel assignment patterns and allows deployment of smaller, non-integer reuse factors based on $\mathrm{C} / \mathrm{I}$ requirements, rather than being restricted within finite values 3,4 , and 7 as determined by (1). In $2 \times(3+1), 3 \times(3+1)$, $4 \times(3+1)$, and $5 \times(3+1)$ reuse plans, we obtain reuse factors of $2.6,4,5.3$, and 6.6 , respectively.

In comparison with theirs conventional counterparts, CAR
TABLE IV

Performancle Comparison Of $7 \times 3$ Vs $5 \times(3+1)$ and $4 \times(3+1)$ ReusePlans

\begin{tabular}{c|c|c|c|c}
\hline $\mathrm{N}$ & Reuse Factor & Worse $\mathrm{C} / \mathrm{I}(\mathrm{dB})$ & Cell Capacity & Increment \\
\hline $7 \times 3$ & 7 & 20.7 & $14.29 \%$ & \\
$5 \times(3+1)$ & 6.6 & 19.3 & $15.00 \%$ & $5.00 \%$ \\
$4 \times(3+1)$ & 5.3 & 17.8 & $18.75 \%$ & $31.25 \%$ \\
\hline
\end{tabular}

TABLE V

Performance Comparison Of $4 \times 3$ Vs. $3 \times(3+1)$ Reuse Plans

\begin{tabular}{c|c|c|c|c}
\hline $\mathrm{N}$ & Reuse Factor & Worse C/I (dB) & Cell Capacity & Increment \\
\hline $4 \times 3$ & 4 & 15.7 & $25.00 \%$ & \\
$3 \times(3+1)$ & 4 & 15.5 & $25.00 \%$ & 0 \\
\hline
\end{tabular}

TABLE VI

Performance Comparison Of $3 \times 3$ Vs. $2 \times(3+1)$ Reuse Plans

\begin{tabular}{c|c|c|c|c}
\hline $\mathrm{N}$ & Reuse Factor & Worse $\mathrm{C} / \mathrm{I}(\mathrm{dB})$ & Cell Capacity & Increment \\
\hline $3 \times 3$ & 3 & 12.3 & $33.33 \%$ & \\
$2 \times(3+1)$ & 2.6 & 11.8 & $37.50 \%$ & $12.50 \%$ \\
\hline
\end{tabular}

reuse plans can increase system capacity up to $31.25 \%$ while still provicle comparable $\mathrm{C} / \mathrm{I}$ margins. The results presented in various papers suggest that reuse plan tighter than $3 \times 3$ is not practical for $100^{\circ}$ to $120^{\circ}$ tri-sectored cellular system, since co-channel cells are adjoined and reducing the separation between co-channel cells hence reduces $\mathrm{C} / \mathrm{I}$ below acceptable level [1][7][8]. CAR $2 \times(3+1)$ has clearly shown otherwise.

Unlike other proposed reuse plans where antennas must be replaced, cells must be realigned, and cluster is large, CAR is simple, and can be implemented in any existing directional antenna system. It truly does not impose any additional cost.

\section{REFERENCES}

[1] L.-C. Wang, "A new cellular architecture based on an interleaved cluster concept," IEEE Transactions on Vehicular Technology, vol. 48, no. 6 , pp. $1809-1818$, Nov. 1999.

[2] H. Tawfik, "Frequency planning considerations for digital cellular systems," IEEE Vehicular Technology Conference, vol. 40, pp. 200206, 1990.

[3] S. Faruque, "Directional frequency reuse for cellular communications," IEEE Personal Wireless Communications, pp. 206-209, 1997.

[4] P. S. Rha, "Frequency reuse scheme with reduced co-channel interference for fixed cellular systems," IEE Electronics Letters, vol. 34 , no. 3, pp. 237-238, Feb. 98.

[5] L.-C. Wang, C. K. Chawla, and L. J. Greenstein, "Performance studies of narrow-beam trisector cellular systems," IEEE Vehicular Technology Conference, vol. 2, pp. $724-730,1998$.

[6] I. Katzela, M. Naghshineh, "Channel assignment schemes for cellular mobile telecommunication systems: a comprehensive survey," IEEE Personal Communications, vol. 3, no. 3, pp. 10-31, June: 1996.

[7] J. Xiang, "A Novel two site frequency reuse plan," IEEEE Vehicular Technology Conference, pp. 441-445, 1996.

[8] Y. Kinıshita, D. Asano, "Enhanced conceptual design formulae for frequency channel double reuse digital systems using sectored cells," IEEE Vehicular Technology Conference, vol. 1, pp. 679-682, 1998.

[9] L.-C. Wang, K. K. Leung, "Performance enhancement in narrow-beam quad-sector cell and interleaved channel assignment in wireless networks," Global Telecommunications Conference, pp. 2719-2724, 1999

[10] CSA Wireless, http://www.csawrls.com 